\title{
Distal hereditary upper limb muscular atrophy
}

\author{
D W Gross, A H Rajput, M Yeung
}

University of

Saskatchewan,

Saskatoon, Sk, Canada

D W Gross

A H Rajput

M Yeung

Correspondence to: $\mathrm{Dr} \mathrm{AH}$ Rajput, Division of

Neurology, Department of Medicine

103 Hospital Drive,

Saskatoon, Sk. S7N 0W8,

Canada. Telephone 001306

966 8007; fax 001306966 8008 .

Received 18 February 1997 and in revised form 3 June 1997

Accepted 9 July 1997
Royal University Hospital,

\begin{abstract}
Objectives-To identify the clinical, electrophysiological, and genetic characteristics of a family with an unusual form of hereditary motor neuron disease.

Methods-Surviving members of a pedigree in which affected members presented with weakness and atrophy of distal musculature in the upper limbs were examined clinically and electrophysiologically, and had genetic testing.

Results-The disease was autosomal dominantly inherited and manifested as weakness and atrophy of distal musculature in the upper limbs, with minimal involvement of lower limbs, brisk reflexes, minimal sensory findings, and considerable variability in severity among the affected persons. Nerve conduction studies disclosed near normal motor conduction velocity, reduced motor compound
\end{abstract}
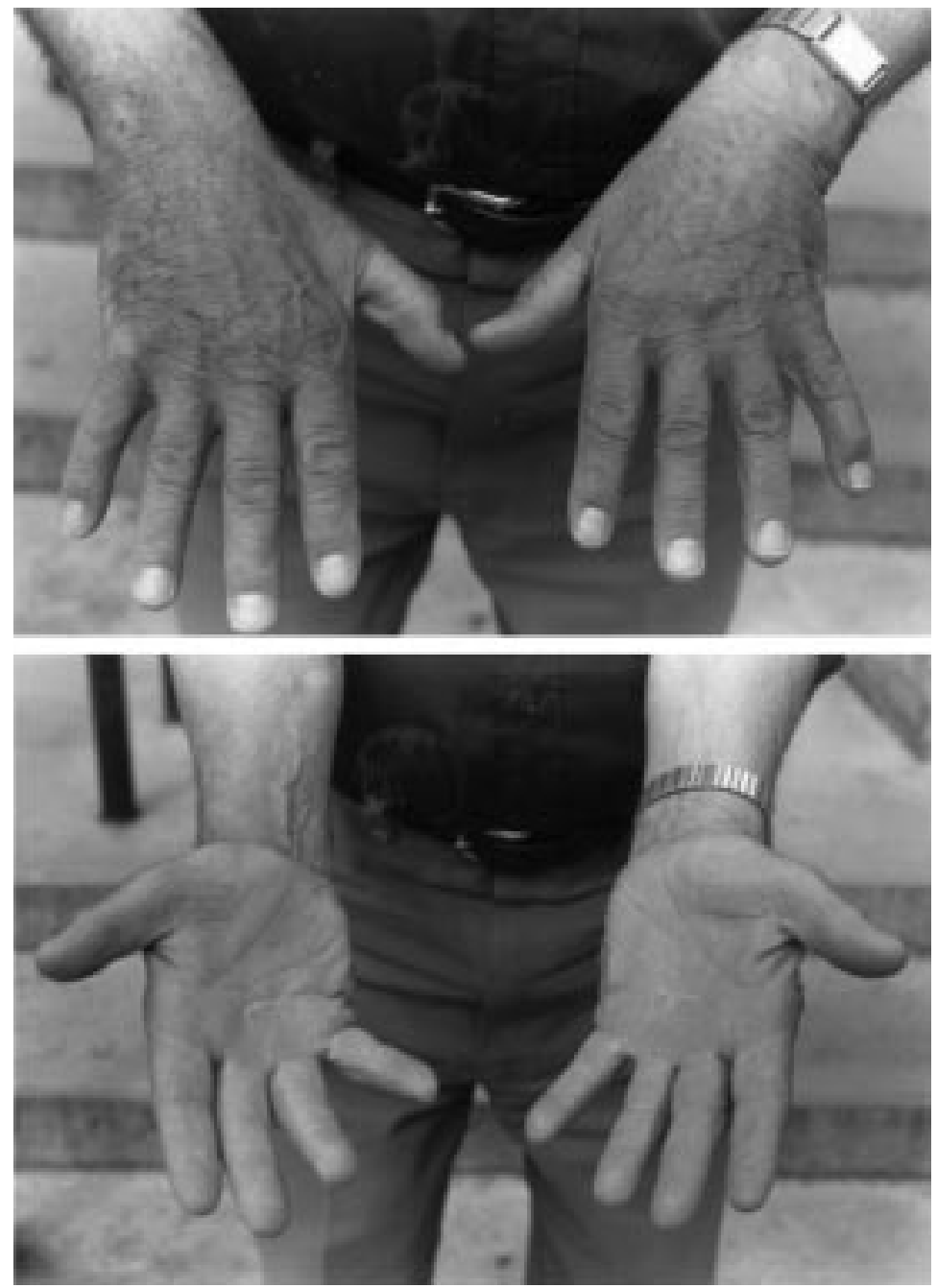

Figure 1 Photographs of patient 6 showing atrophy of all intrinsic hand muscles action potential amplitude, prolonged distal motor latency, prolonged sensory latency, and normal sensory compound action potential amplitude. Needle electrode examination showed reduced number and increased size of motor unit potentials, but no fibrillations or fasciculations.

Conclusion- Distal upper limb muscular atrophy is a distinct clinical entity.

(f Neurol Neurosurg Psychiatry 1998;64:217-220)

Keywords: hereditary; motor neuron disease

The syndrome of hereditary peroneal muscular atrophy can be caused by different diseases. These include hereditary motor and sensory neuropathy (HMSN), hereditary distal spinal muscular atrophy or hereditary motor neuronopathy (HMN), and chronic hereditary amyotrophic lateral sclerosis (ALS). A few families have been reported with familial motor neuron disease affecting the distal muscles of the upper limbs, with minimal involvement of the lower limbs. ${ }^{1-6}$ The current study is an attempt to define clinical, electrophysiological, and genetic characteristics of a hereditary condition in a large kindred which is manifest by weakness and atrophy affecting the distal muscles of the upper limbs.

\section{Case report}

A 63 year old man presented for investigation of gait disturbance. He had been diagnosed as having "Parkinson's disease" three years previously and had been started on antiparkinsonian medication with no improvement. Along with the gait difficulties, he also complained of progressive weakness and atrophy in both hands, from childhood. He had a 10 year history of non-insulin dependent diabetes mellitus. Family history was relevant in that family members in four consecutive generations were affected similarly with weakness and wasting of hand muscles.

General physical examination was unremarkable. Neurological examination disclosed normal sensorium and normal cranial nerves. There was evidence of mild severity parkinsonism. He had pronounced symmetric atrophy of both median and ulnar innervated hand muscles (fig 1) and moderate to severe weakness of involved muscles, pes cavus deformity, and mild to moderate weakness of distal leg muscles. Deep tendon reflexes were brisk bilaterally in both upper and lower limbs and plantar responses were up going bilaterally. Sensory examination was normal, as was coordination. 


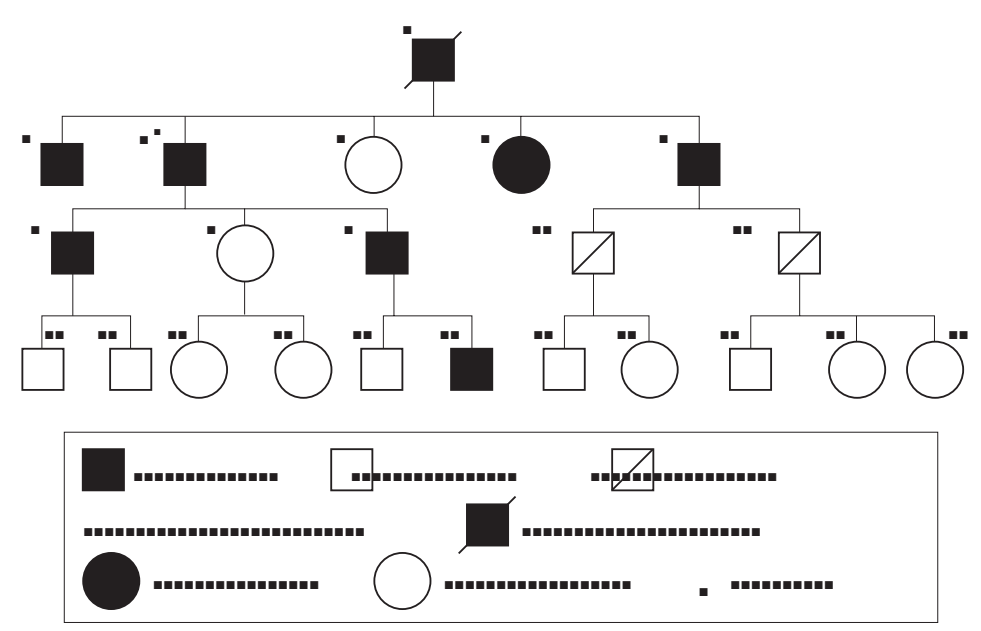

Figure 2 Pedigree with distal ulnar-median muscular atrophy

Motor nerve conduction studies showed extremely small compound action potential amplitudes in the upper limbs with prolonged distal latencies, normal conduction velocities, and low compound action potential amplitudes and slow conduction velocity in the lower limbs. There was mild prolongation of sensory latencies. Needle electrode examination showed no fibrillations or fasciculations and a decreased number and increased size of motor units. Genetic testing for HMSN type 1A was negative.

The patient was diagnosed as having a hereditary motor neuron disease and mild Parkinson's disease.

\section{Methods}

Surviving members of the propositus's family had clinical, electrophysiological, and genetic testing after informed consent was obtained. All family members received a detailed history and physical examination by the same examiner. Motor nerve conduction studies and needle electrode examinations were performed using either a Nicolet-Viking II or a Nihon Kohden-Neuropack 4 Mini EMG machine. Conventional orthodromic motor and antidromic sensory nerve conduction studies were performed using surface electrodes for stimulating and recording. Clinically directed needle EMG examinations were performed. Insertional activity, spontaneous activity, motor unit potential configuration, and recruitment pattern were recorded. Analysis for the duplication in chromosone $17 \mathrm{p} 11.2-12$ was carried out as previously described.

\section{Results}

Twenty one members of the family were examined (fig 2). The only family member who was not examined was the deceased patriarch (patient 1). A total of 15 patients had electrophysiological studies. Of the six patients who did not, three were young children, and three were not able to come to the laboratory for testing. Blood samples were originally taken from the propositus and sent to Genica laboratories for investigation of HMSN 1A, which was negative. Subsequently, blood samples were collected from all patients, except patient 22 (who was a newborn infant at the time the study was undertaken) and are currently being investigated for a genetic abnormality.

Clinical evaluation identified eight members (including the deceased patriarch who was positive by history) as being phenotypically affected (fig 2). They were present in four consecutive generations and included males and females with apparent complete penetrance. Male to male transmission was present, confirming autosomal dominant inheritance. From available information on the extended family, neither the affected patriarch's (patient 1) parents, siblings, nor any other relatives expressed the phenotype. All affected cases first became aware of deficit in childhood or early adolescence noting hand weakness and wasting as the most prominent presenting symptoms. Of the affected members, patient 9 was thought to be clinically normal, but had significant abnormalities on neurological examination. Patients 2 and 5 were mildly symptomatic, and patients $3,6,7$, and 15 were more severely affected. In the affected patients, the deficits did not impair their ability to work and two of the four more severely affected members were manual labourers. Affected members showed slow progression of symptoms.

All affected members had symmetric weakness of the distal muscles of the upper limbs in the distribution of the radial, ulnar, and median nerves. The weakness varied in severity between patients from mild $(4+/ 5)$ to severe (3/5). Only one affected patient (2) did not show evidence of muscle atrophy, whereas all others showed symmetric atrophy of the thenar and hypothenar eminences as well as the dorsal interossei. All affected members had brisk reflexes in upper and lower limbs, in comparison to unaffected family members. Four affected members had equivocal plantar responses $(3,5,6$, and 7$)$, with plantar responses being flexor in all other members. Three patients $(3,6$, and 15) also had distal weakness in the lower limbs. Only patient 6 had clinically identifiable sensory loss to pin prick in a stocking distribution. He had no history of diabetes mellitus, and no other cause of sensory dysfunction could be identified. Only the propositus had pes cavus deformity.

Electrophysiological studies were done on both affected and unaffected members. The table gives the results. Mean median and ulnar nerve conduction velocities and amplitudes were significantly greater (with the exception of median nerve conduction velocity, which approached significance $(\mathrm{p}=0.08))$, and the median and ulnar nerve motor and sensory distal latences latencies were significantly less in the unaffected members Sensory action potential amplitudes were essentially the same in both affected and unaffected members. Needle electrode examinations of affected members consistently showed no evidence of fibrillations or fasciculations and motor units were reduced in number but increased in amplitude.

Two members who had normal neurological examinations were found to have some abnor- 
Table 1 Nerve conduction studies for affected and unaffected people

\begin{tabular}{llll}
\hline & Affected people & Unaffected people & p Value \\
\hline Median nerve conduction velocities (m/s) & 56 & 60.1 & 0.08 \\
Ulnar nerve conduction velocities (m/s) & 53.7 & 61.9 & 0.04 \\
Median nerve CMAP amplitudes (mV) & 7.99 & 12.3 & 0.01 \\
Ulnar nerve CMAP amplitudes (mV) & 8.11 & 11.4 & 0.003 \\
Median nerve motor distal latencies (ms) & 5.22 & 3.09 & 0.04 \\
Ulnar nerve motor distal latencies (ms) & 3.35 & 2.42 & 0.003 \\
Median nerve sensory distal latencies (ms) & 3.58 & 2.81 & 0.02 \\
Ulnar nerve sensory distal latencies (ms) & 3.17 & 2.5 & 0.002 \\
\hline
\end{tabular}

malities on electrophysiological testing. Patient 10 (aged 41) showed prolonged median motor distal latency at $4.68 \mathrm{~ms}$ and reduced median and ulnar motor compound action potential amplitudes at $4.3 \mathrm{mV}$ and $4.73 \mathrm{mV}$ respectively. Patient 11 (aged 38) showed median motor distal latencies of $4.28 \mathrm{~ms}$ and $4.0 \mathrm{~ms}$ and median sensory amplitudes of $13.5 \mu \mathrm{V}$ and $18.8 \mu \mathrm{V}$.

\section{Discussion}

We have studied a pedigree which has an autosomal dominant syndrome of motor neuronopathy. This disorder may have resulted from spontaneous mutation at patient 1 . In this pedigree, eight members were definitely affected, based on history, physical examination, and abnormal electrophysiological studies. An additional two members were probably affected, based on abnormal electrophysiological studies alone. This disorder shows complete penetrance with considerable variability in severity and clinical findings among affected members. Affected members show progressive weakness and atrophy of intrinsic hand muscles, with brisk reflexes, and, in some cases, equivocal plantar responses. Severely affected members may have weakness in the lower limbs, and one severely affected member also has sensory abnormalities. Electrophysiological testing showed normal motor conduction velocities, prolonged motor distal latencies, reduced motor amplitudes, and prolonged sensory latencies, with no spontaneous muscle activity and reduced number and increased size of motor units. The physical findings along with the electrophysiological results indicate involvement of the corticospinal tract, as well as peripheral motor nerves or anterior horn cells.

The differential diagnosis of this syndrome would be similar to that of hereditary peroneal muscular atrophy in that it would include HMSN, HMN, and familial amyotrophic lateral sclerosis. Harding and Thomas, in their review of 262 cases of peroneal muscular atrophy divided their patients into three categories: demyelinating HMSN (type I), neuronal HMSN (type II), and HMN. ${ }^{9}$ The criteria for diagnosis of HMSN I was median motor conduction velocities less than $38 \mathrm{~m} / \mathrm{s}$. The differentiation between HMSN II and HMN, in this study, was based on the presence or absence of sensory findings and abnormal sensory nerve action potentials. Harding and Thomas excluded complex syndromes such as familial spastic paraplegia; however, from Dyck et al, this would be classified as HMSN V. ${ }^{10}$ Hereditary ALS is a disease of the upper and lower motor neurons that can vary greatly in age of onset, rate of progression, and ultimate severity of disease. Hamida et al reviewed 43 patients from 17 families with chronic ALS of juvenile onset, all of whom had no sensory findings and normal sensory nerve conduction studies. ${ }^{11}$ The patients in this review were divided into three groups: patients with upper limb atrophy with or without bulbar findings, spastic paraplegia with peroneal muscular atrophy, and spastic pseudobulbar palsy with spastic paraplegia.

By these criteria, our pedigree does not fit into HMSN I as all the affected members had near normal motor conduction velocities, although the prolonged motor and sensory latencies suggest demyelination. In the review of Harding and Thomas, $73 \%$ of patients with HMSN II had absent median sensory nerve action potentials, whereas these were reduced in the other $27 \% .{ }^{8}$ One patient in our pedigree had sensory findings, and sensory distal latencies were reduced in affected members; however, sensory action potentials were of normal amplitude. HMN is typically a disease of the lower motor neurons, as opposed to ALS which can affect both upper and lower motor neurons. The presence of both upper and lower motor neuron findings in our pedigree would favour the diagnosis of a chronic hereditary form of ALS. However, the finding of sensory loss in one member, along with abnormal sensory and motor distal latencies would be against this diagnosis. Although ALS is considered to be a disease confined to the motor system, clinical and pathological studies have shown involvement of other structures, including the posterior columns and peripheral sensory axons in both sporadic and familial forms of the disease. ${ }^{1213}$

Several other pedigrees with hereditary neuropathies have been reported in whom the intrinsic hand muscles are the only, or the most severely, affected muscles. Other workers have reported pedigrees with motor neuropathies predominantly, or solely, affecting the upper limbs. ${ }^{1-6}$ All of these pedigrees, except that described by Meadows and Marsden, ${ }^{2}$ were reported to have upper neuron findings. Only our pedigree and that described by Van Gent $e t$ $a l^{5}$ showed evidence of sensory nerve involvement. Only our pedigree had abnormal sensory and motor distal latencies. All the pedigrees except that described by Cross and McKusick $^{6}$ showed autosomal dominant inheritance (the pedigree described by Cross and McKusick showed autosomal recessive inheritance). Although some differences among the pedigrees exist, the similarities between them is striking, and it seems likely that they do represent a common phenotype, even though the different pedigrees had been classified in the past as HSMN, HMN, and chronic juvenile ALS. ${ }^{9-11}$ Further study of this and other similar pedigrees is necessary to better understand what genetic mutation leads to this phenotype, whether the same mutation is responsible for all forms of neuropathies affecting upper limbs, and the reason why some hereditary neuron- 
opathies preferentially affect motor as opposed to sensory nerves.

We acknowledge JR Donat and Dr Phillip F Chance.

1 Lander CM, Eadie MJ, Tyrer JH. Hereditary motor peripheral neuropathy predominantly affecting the arms. $\mathscr{F}$ Neurol Sci 1976;28:389-94.

2 Meadows JC, Marsden CD. A distal form of chronic spinal

muscular atrophy. Neurology 1969;19:53-8.
3 O'Sullivan PJ, McLeod JG. Distal chronic spinal muscular atrophy involving the hands. $\mathcal{F}$ Neurol Neurosurg Psychiatry 1978;41:653-8.

4 Silver JR. Familial spastic paraplegia with amyotrophy of the hands. F Neurol Neurosurg Psychiatry 1966;29:135-44.

5 Van Gent E, Hoogland RA, Jennekens FGI. Distal amyotrophy of predominantly the upper limbs with pyramidal feaphy of predominantly the upper limbs with pyramidal fea-
tures in a large kinship. $\mathcal{F}$ Neurol Neurosurg Psychiatry 1985; 48:266-9.

6 Cross HE, McKusick VA. The Troyer syndrome, a recessive form of spastic paraplegia with distal muscle wasting. Arch 67; 16:473-85.

7 Lupski JR, Chance PF, Garcia CA. Inherited primary peripheral neuropathies, molecular genetics and clinical Implications of CMT1A and HNPP. $\mathscr{f} A M A$ 1993;270:2326-30
8 Harding AE, Thomas PK. The clinical features of hereditary motor and sensory neuropathy types I and II. Brain 1980;103:259-80.

9 Harding AE, Thomas PK. Hereditary distal spinal muscular atrophy. I Neurol Sci 1980;45:337-48.

10 Dyck PJ, Chance P, Lebo R, Carney JA. Hereditary motor and sensory neuropathies. In: Dyck PJ ed. Peripheral neuropathy, 3rd ed. Philadelphia: WB Saunders, 1993;57: neuropathy

11 Ben Hamida M, Hentati F, Ben Hamida C. Hereditary motor system diseases (chronic juvenile amyotrophic lateral sclerosis). Brain 1990;113:347-63.

12 Bradley WG, Good P, Rasool CG, Adelman LS. Morphometric and biochemical studies of peripheral nerves in amyotrophic lateral sclerosis. Ann Neurol 1983;14:267-77.

13 Horton WA, Eldridge R, Brody JA. Familial motor neuron disease, evidence for at least three different types. Neurology 1976;26:460-5.

14 Harding AE. Inherited neuronal atrophy and degeneration predominantly of lower motor neurons. In: Dyck PJ ed. Peripheral neuropathy, 3rd ed. Philadelphia: WB Saunders, 1993;55:1051-63.

15 Harding AE, Thomas PK. Peroneal muscular atrophy with pyramidal features. I Neurol Neurosurg Psychiatry 1984;47: $168-72$. 